\title{
Gamow shell-model calculations of drip-line oxygen isotopes
}

\author{
K. Tsukiyama, ${ }^{1}$ M. Hjorth-Jensen, ${ }^{2}$ and G. Hagen ${ }^{3}$ \\ ${ }^{1}$ Department of Physics, University of Tokyo, 7-3-1 Hongo, Bunkyo-ku, Tokyo, Japan \\ ${ }^{2}$ Department of Physics and Center of Mathematics for Applications, University of Oslo, N-0316 Oslo, Norway \\ ${ }^{3}$ Physics Division, Oak Ridge National Laboratory, \\ P.O. Box 2008, Oak Ridge, Tennessee 37831, USA
}

(Dated: October 29, 2018)

\begin{abstract}
We employ the Gamow shell model (GSM) to describe low-lying states of the oxygen isotopes ${ }^{24} \mathrm{O}$ and ${ }^{25} \mathrm{O}$. The many-body Schrödinger equation is solved starting from a two-body Hamiltonian defined by a renormalized low-momentum nucleon-nucleon (NN) interaction, and a spherical Berggren basis. The Berggren basis treats bound, resonant, and continuum states on an equal footing, and is therefore an appropriate representation of loosely bound and unbound nuclear states near threshold. We show that such a basis is necessary in order to obtain a detailed and correct description of the low-lying $1^{+}$and $2^{+}$excited states in ${ }^{24} \mathrm{O}$. On the other hand, we find that a correct description of binding energy systematics of the ground states is driven by proper treatment and inclusion of many-body correlation effects. This is supported by the fact that we get ${ }^{25} \mathrm{O}$ unstable with respect to ${ }^{24} \mathrm{O}$ in both oscillator and Berggren representations starting from a ${ }^{22} \mathrm{O}$ core. Furthermore, we show that the structure of these loosely bound or unbound isotopes are strongly influenced by the ${ }^{1} S_{0}$ component of the NN interaction. This has important consequences for our understanding of nuclear stability.
\end{abstract}

PACS numbers: 21.10.-k, 21.30.-x, 21.60.-n, 24.30.Gd, 27.30.+t

Introduction. The study of nuclei far from stability is a leading direction in nuclear physics, experimentally and theoretically. It represents a considerable intellectual challenge to our understanding of the stability of matter itself, with potential implications for the synthesis of elements. An important aspect of this research direction is to understand how magic numbers and shells appear and evolve with increasing numbers of neutrons or protons. Except for a qualitative understanding of the neutron and proton dependence of the magic numbers in terms of mean-field models, we lack a quantitative theoretical understanding in terms of the basic constituents of the underlying nuclear many-body Hamiltonian.

For mesoscopic systems like nuclei, the interpretation of the standard magic numbers $2,8,20,28,50,82$, and 126 is linked to the interplay between the filling up of single-particle (s.p.) orbitals (s.p. picture) and the underlying interactions. Magic numbers lead to so-called shell gaps in the s.p. spectra near the Fermi energy. For various magic numbers, in particular for stable doubly magic nuclei, one can have a large number of stable isotopes (increasing number of neutrons $N$ but fixed number of protons $Z$ ) or isotones (increasing number of protons $Z$ and fixed $N$ ). Eventually, as one adds more neutrons or protons, viz., moving away from the valley of nuclear stability towards the drip lines, the outermost nucleons literally start to drip off the nuclei, thereby defining the very limits of stable matter.

Nuclei near the drip line are open-quantum manybody systems for which the coupling with the scattering continuum plays an important role, and should therefore be explicitly taken into account. Configuration interaction (shell-model) methods, such as the Gamow shell model (GSM) (see Ref. [1] for a recent review of the GSM) or the continuum shell model [2], have been developed in order to properly include the coupling with the scattering continuum. The inclusion of continuum states complicates the solution of the many-body problem considerably, as the number of many-body basis states will explode. This has therefore motivated different approaches to the description of loosely bound and unbound nuclear states, such as the Density-Matrix-Renormalization-Group (DMRG) 3] and Coupled-Cluster approaches [4]. Furthermore, to complicate the solution strategies of the nuclear manybody problem is the need to include three-nucleon forces (3NFs). Recent results for light nuclei using $a b$ initio methods such as the Green's function Monte-Carlo (GFMC) [5] and the no-core shell model (NCSM) [ 6], demonstrate that $3 \mathrm{NFs}$ are needed in order to give the correct binding energies and spectroscopy when comparing to experiment. It is still an open research problem what the role of $3 \mathrm{NF}$ s is in medium mass and neutronrich nuclei close to the drip line. This applies also to our understanding of how different parts of the underlying nucleon-nucleon $(\mathrm{NN})$ interaction, such as the spin-orbit force [7, [8] and the tensor force [9, 10], affect the structure of nuclei close to the drip line.

In this work we study the ground- and low-lying states of ${ }^{24} \mathrm{O}$ and ${ }^{25} \mathrm{O}$ within the GSM framework. We derive for the first time a realistic effective shell-model interaction that includes the effect of the scattering continuum. The effective interaction is derived using many-body perturbation theory (MBPT) starting with a Hamiltonian which reproduces NN scattering data. The choice of the oxygen isotopes is motivated by several reasons. First, the oxygen isotopes are the heaviest isotopes for which the drip line is well established. There are large exper- 
imental campaigns worldwide [11, 12, 13] which aim at uncovering the properties of the oxygen isotopes, both at or close to the drip line and beyond. Two out of four stable even-even isotopes exhibit a doubly magic nature, namely ${ }^{22} \mathrm{O}(Z=8, N=14)[14]$ and ${ }^{24} \mathrm{O}(Z=8, N=16)$ [15, 16]. The isotopes ${ }^{25-28} \mathrm{O}$ are all believed to be unstable towards neutron emission, even though ${ }^{28} \mathrm{O}$ is a doubly magic nucleus within the standard shell-model picture. Secondly, many-body descriptions starting with effective two-body Hamiltonians defined for a model space comprising the $1 s_{1 / 2}, 0 d_{5 / 2}$, and $0 d_{3 / 2}$ s.p. orbitals, have always defied a proper reproduction of the experimental data [18, 19, 20]. To remedy this, the two-body shell-model interaction is usually fitted (fully or partially) [2, 18, 19, 21] to reproduce experimental data for nuclei in the range $16<A<40$. Without fitting the two-body Hamiltonians, which are typically derived using MBPT, shell-model calculations of the oxygen isotopes fare reasonably up to ${ }^{20} \mathrm{O}$, but produce too compressed spectra in ${ }^{22} \mathrm{O}$ and lead to neutron separation energies that are always positive up to ${ }^{28} \mathrm{O}$. All isotopes ${ }^{17-28} \mathrm{O}$ that can be reached via this model space result as well bound, in clear contradiction with experiment. These results pertain to all available models of the NN interaction [18, 20] within the above model space. The ground states and excited states of the odd-even isotopes are also poorly reproduced (see for example Refs. [18, 19]).

Recent ab-initio coupled-cluster calculations of the oxygen isotopes [22] showed that chiral NN interactions can produce a rather flat binding energy curve of the oxygen isotopes ranging from ${ }^{24} \mathrm{O}$ to ${ }^{28} \mathrm{O}$. It was illustrated that the existence of ${ }^{28} \mathrm{O}$ cannot be ruled out from $a b$ initio theory starting from modern chiral interactions. It was concluded that $3 \mathrm{NFs}$ will eventually decide the matter. Recent shell-model calculations of the oxygen isotopes within the $s$ - $d$ shell showed that inclusion of effective $3 \mathrm{NFs}$ give added repulsion in the heavier oxygen isotopes and resulted in an unstable ${ }^{28} \mathrm{O}[23$.

Obviously, the understanding of the evolution of binding energies and the location of the drip line in the oxygen isotopes are still unsolved theoretical problems. As shown in Refs. 22, 23], proper treatment of many-body effects are crucial in the oxygen isotopes. However, it is still an open issue what the role of the continuum is in the evolution of shell structure and in a correct description of binding energy systematics in neutron-rich oxygen isotopes. As found in Ref. [24], a proper treatment of the continuum was necessary in order to explain the parity inversion of the ${ }^{11} \mathrm{Be}$ ground state. It is therefore important to investigate what the role of continuum is on ground and excited states in neutron-rich oxygen isotopes. It is the aim of this article to throw light on, and disentangle the different effects coming from manybody correlations and the proximity of the continuum on the evolution of binding energies and shell structure in neutron-rich oxygen isotopes. Further, we will investigate how different components of the NN interaction influence the low-lying resonance states in drip-line oxy- gen isotopes.

Method, interaction, and model space. In this work we use the GSM to describe the low-lying states in ${ }^{24} \mathrm{O}$ and the ground state of ${ }^{25} \mathrm{O}$. We construct the effective shellmodel interaction starting from ${ }^{22} \mathrm{O}$ as a closed-shell core and use MBPT through second order [20]. The isotope ${ }^{22} \mathrm{O}$ has been found to be a closed-shell nucleus with a considerable shell gap, making it suitable as a starting point for shell-model calculations. With ${ }^{22} \mathrm{O}$ as a closedshell core we have at most three valence particles, making effects coming from a proper treatment of the continuum more transparent. So far, the effective interaction has typically been constructed using oscillator states as intermediate particle states. In this work, we investigate for the first time what the role of the continuum is in constructing a realistic effective shell-model interaction and in describing spectra of neutron-rich oxygen isotopes.

The major advantage of the GSM is that it unifies structure and reaction properties of nuclei, that is, bound and scattering degrees of freedom are treated equally. Nuclei close to the scattering threshold have low-lying states that are in the continuum, and the GSM provides a simple framework for calculating the energy and lifetimes of these states. The starting point of the GSM is the Berggren completeness relation [28]. This s.p. basis treats bound, resonant, and scattering states on an equal footing, and it is the basic idea of the GSM to expand the many-body wave function in Slater determinants built from this basis. The representation of the Hamiltonian will no longer be Hermitian but rather complex symmetric, and a diagonalization will yield bound state spectra in addition to resonant state spectra. Hence, the energy and lifetime of the many-body resonant state comes out directly from the approach.

In constructing the s.p. Berggren basis, we start from the one-body Hamiltonian for a spherical Woods-Saxon (WS) potential (see for example Ref. [25]). The Berggren basis is obtained by diagonalizing the one-body Hamiltonian in a plane wave basis defined on a deformed contour $L^{+}$in the complex momentum plane (see Ref. [27] for details). This method is also known as the ContourDeformation-Method. There is freedom in choosing the contour $L^{+}$provided the Schrödinger equation can be analytically continued in the complex momentum plane. Fully converged results should be independent of the choice of the contour, and in the following we used two different contours, $L_{1}^{+}$and $L_{2}^{+}$, as given in Ref. [27], to check that our results are converged. The parameters of the WS potential are given by $V_{0}=55 \mathrm{MeV}, V_{\text {so }}=29$ $\mathrm{MeV}, R=3.1 \mathrm{fm}$, and $d=0.45 \mathrm{fm}$, where the shorthand $V_{\text {so }}=29$ stands for the spin-orbit term. These parameters are chosen such that the energies of the s.p. orbitals $\nu 0 d_{5 / 2}, \nu 1 s_{1 / 2}$, and $\nu 0 d_{3 / 2}$ are close to the experimental values obtained by taking the binding energy differences of ${ }^{21} \mathrm{O}_{-}{ }^{22} \mathrm{O}$ and ${ }^{23} \mathrm{O}_{-}{ }^{22} \mathrm{O}$. We obtain, in units of $\mathrm{MeV}, \epsilon_{\nu 0 d 5 / 2}=-6.53(-6.85), \epsilon_{\nu 1 s 1 / 2}=-2.73(-2.74)$ and $\epsilon_{\nu d 3 / 2}=1.43-0.01 i(1.26-0.2 i)$, where numbers in parenthesis are experimental values. We refer hereafter to this 
basis as a WS Gamow basis. In our shell-model study of the oxygen isotopes ${ }^{24} \mathrm{O}$ and ${ }^{25} \mathrm{O}$ we will use the WS Gamow basis for the neutron partial waves $s_{1 / 2}, d_{3 / 2}$, and $d_{5 / 2}$, while for protons and all other partial waves, we use the oscillator representation.

In constructing the effective shell-model interaction for neutron-rich oxygen isotopes, we start from the intrinsic nuclear Hamiltonian $H=t-t_{\mathrm{CoM}}+V$, where $t$ denotes the kinetic energy, and $t_{\mathrm{CoM}}$ is the kinetic energy of the center of mass. For the interaction $V$, we employ the NN interaction by Entem and Machleidt [29, 30] derived from chiral effective field theory (EFT) at nextto-next-to-next-to-leading order $\left(\mathrm{N}^{3} \mathrm{LO}\right)$ and with a 500 $\mathrm{MeV}$ cutoff. This interaction still has considerable highmomentum components, making MBPT non-convergent. In order to construct the effective shell-model interaction $V_{\text {eff }}$ using MBPT, we use a low-momentum interaction $V=V_{\text {low }-k}$, obtained by integrating out those momentum components above a certain cutoff $\Lambda[26]$. Thereafter we generate $V$ in the WS Gamow basis using the procedure outlined in Ref. 27]. It is well known that this renormalization induces forces of higher rank. In a completely renormalized theory, all observables are independent on the cutoff $\Lambda$, and therefore the dependence of our results on the cutoff $\Lambda$ tells us about the missing many-body physics. In this work we start from a ${ }^{22} \mathrm{O}$ core and limit ourselves to two-body effective shell-model interactions only. Since we have at most three neutrons outside the closed-shell core, it is reasonable to expect that the effect coming from effective three-body forces is small.

In order to proceed with the construction of the effective shell-model interaction, we need to define a model space for the valence neutrons. Since we will perform our GSM calculations starting with ${ }^{22} \mathrm{O}$ as a closed shell core, i.e., the $0 d_{5 / 2}$ shell is filled, our many-body states are constructed by letting all valence neutrons act in the model space defined by the the s.p. orbitals $1 s_{1 / 2}$ and $i d_{3 / 2}$, where $i=0,1, \ldots$ denote the $i$-th discretized continuum state for the $d_{3 / 2}$ partial wave. The effect of the continuum from the $s_{1 / 2}$ and $d_{5 / 2}$ partial waves are taken into account through the construction of the effective interaction. The effective shell-model interaction $V_{\text {eff }}$ is then constructed for this model space using MBPT [20],

$$
H_{e f f}=\hat{Q}-\hat{Q}^{\prime} \int \hat{Q}+\hat{Q}^{\prime} \int \hat{Q} \int \hat{Q}-\cdots .
$$

Here $\hat{Q}(\omega)$ is given by diagrams which are valence-linked and irreducible. The argument $\omega$ is the starting energy, which is the sum of the unperturbed s.p. energies. The object $\hat{Q}(\omega)$ is defined as

$$
\hat{Q}(\omega)=P H_{1} P+P H_{1} Q \frac{1}{\omega-Q H Q} Q H_{1} P .
$$

In this work we take into account diagrams up through second order in perturbation theory in order to construct $\hat{Q}(\omega)$. In Fig. 1 the diagrams that contribute up to second order in the effective interaction are shown. The

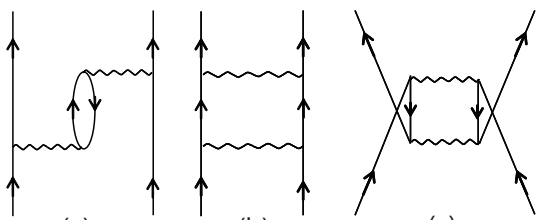

(a)

(b)

(c)

FIG. 1: The second-order contributions to $V_{\text {eff }}$.

intermediate s.p. states in each diagram in can be either resonant, non-resonant continuum, or bound states. In our calculations we neglect the contributions from folded diagrams; see Ref. [20] for further details.

Results. We now turn to the GSM calculations of lowlying states in ${ }^{24} \mathrm{O}$ and ${ }^{25} \mathrm{O}$. For the case of ${ }^{24} \mathrm{O}$ we have two neutrons outside the ${ }^{22} \mathrm{O}$ core, and we express the ground state of ${ }^{24} \mathrm{O}$ by filling the $1 s_{1 / 2}$ orbital. This orbital is well bound, and with a neutron separation energy of $S_{n}=2.7 \mathrm{MeV}$ for ${ }^{23} \mathrm{O}$ [31]. We therefore expect mixing of higher-lying states to be small in the ground state of ${ }^{24} \mathrm{O}$, and we take this effect into account through the construction of the effective interaction using manybody perturbation theory. For the excited states $J=1$ and $J=2$ of ${ }^{24} \mathrm{O}$, we expand the wave function in a set of basis states $\left|i J^{+}\right\rangle=\left|1 s_{1 / 2} \otimes i d_{3 / 2} ; J^{+}\right\rangle$. The orbital $i d_{3 / 2}$ denote the $i$-th discretized s.p. state of the WS Gamow basis, and $i=1, \cdots n_{\text {gamow }}$, where $n_{\text {gamow }}$ is typically between 20 and 35 . In our calculations we used $n_{\text {gamow }}=30$, and we checked that this was sufficient to reach convergence.

In Fig. 22 the excitation energies of the low-lying states in ${ }^{24} \mathrm{O}$ and the ground state in ${ }^{25} \mathrm{O}$ with respect to the ground state of ${ }^{24} \mathrm{O}$ are shown. In these calculations we employed a cutoff $\Lambda=2.1 \mathrm{fm}^{-1}$ for the $V_{\text {low }-k}$ interaction. We have varied the cutoff $\Lambda$ around $2.1 \mathrm{fm}^{-1}$ and found that our results are nearly independent of the cutoff $\Lambda$, meaning that the effect of neglected three- and many-body forces are small and will not change our conclusions. Our GSM calculations give energies for the low-lying states that compare well with the experimental values. The splitting between the $1^{+}$and the $2^{+}$states of ${ }^{24} \mathrm{O}$ compares well with experiment. The theoretical spacing obtained with our GSM approach is $540 \mathrm{keV}$, while the corresponding experimental value is $600 \mathrm{keV}$. Our calculated widths for the $1^{+}$and $2^{+}$resonant states are 0.44 and $0.26 \mathrm{MeV}$, respectively. In Ref. [13], Hoffman reported that the widths are $0.03_{-0.03}^{+0.12} \mathrm{MeV}$ for the $1^{+}$state and $0.05_{-0.05}^{+0.21} \mathrm{MeV}$ for the $2^{+}$state. Our calculations overestimate the width of the $1^{+}$state as compared to experiment, on the other hand our calculated width of the $2^{+}$state is within the experimental uncertainties. As a comparison, we show results obtained with other effective interactions. The new universal $s d$ Hamiltonians (USDA and USDB), obtained by revising the USD interaction in order to be more suitable for exotic nuclei [19], results in a good agreement with experimental data. However, one should note that some effects coming 
from the continuum and 3NFs are implicitly included in the new USD two-body matrix elements due to the fit made to experimental data. Although the USD interaction reproduces nicely two of the three experimental data points, the $2^{+}$state in ${ }^{24} \mathrm{O}$ comes out as a bound excited state, a fact which disagrees with recent experiments. We also show results obtained with a pure harmonic oscillator basis. In this case, the discretized continuum is not included in the model space, and we construct the effective interaction using perturbation theory through second order (see Fig. 1), and a model space consisting of neutrons in the orbitals $\left(1 s_{1 / 2}, 0 d_{3 / 2}\right)$. Using a model space consisting of only bound oscillator orbitals puts the $1^{+}$and $2^{+}$states in ${ }^{24} \mathrm{O}$ too high in energy when comparing with experiment. With the inclusion of the continuum in the model space, the $1^{+}$and $2^{+}$states get lower in energy and also closer to experiment. This clearly shows an effect coming from a proper treatment of the continuum, and should therefore be included in a realistic description of low-lying states in ${ }^{24} \mathrm{O}$. Further, the $2^{+}$state in ${ }^{24} \mathrm{O}$ is a resonant state and cannot be properly described in an oscillator basis.

Turning to the case of the ground state of ${ }^{25} \mathrm{O}$, our GSM calculations correctly predict that it is particle unstable, but puts it at a higher energy $(E=2.1, \Gamma=0.5$ $\mathrm{MeV})$ as compared to recent experimental data $(E=0.77$, $\Gamma=0.17 \mathrm{MeV})$. To get a better description of this state, configurations like $\nu\left(1 s_{1 / 2}\right)\left(i d_{3 / 2}\right)^{2}$ should be included since the GSM is currently solved in a filling configuration. Again we compare our GSM result for ${ }^{25} \mathrm{O}$ with

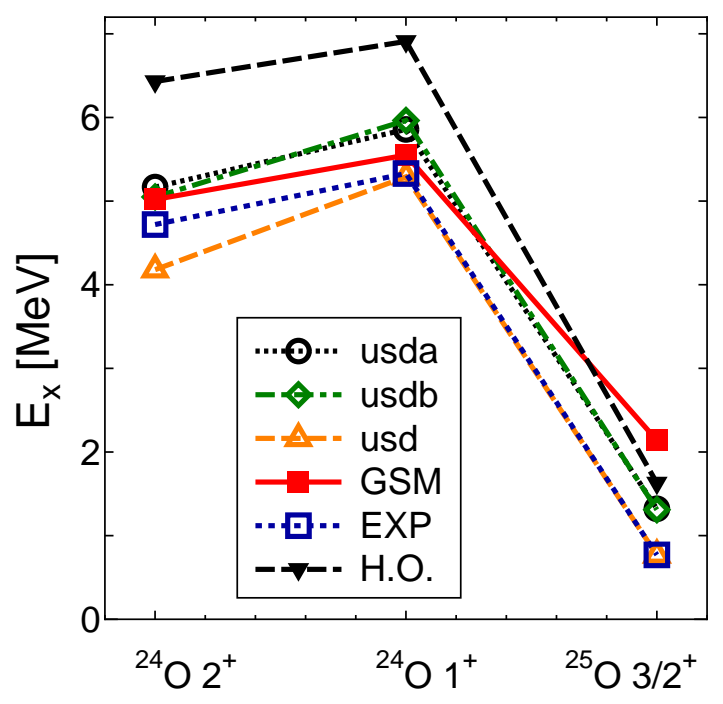

FIG. 2: The excitation energies of the $J^{\pi}=1^{+}$and $J^{\pi}=2^{+}$ states in ${ }^{24} \mathrm{O}$ and the ground-state energy of ${ }^{25} \mathrm{O}$ measured from the ground states in ${ }^{24} \mathrm{O}$.

a shell-model calculation where only the bound oscillator orbitals $\left(1 s_{1 / 2}, 0 d_{3 / 2}\right)$ define the model space. An important point to note is that ${ }^{25} \mathrm{O}$ comes out higher in energy as compared to ${ }^{24} \mathrm{O}$, reflecting that it is unstable towards neutron emission. Even though the oscillator basis cannot provide us with a width or a lifetime for the ground state of ${ }^{25} \mathrm{O}$, it predicts ${ }^{25} \mathrm{O}$ as particle unstable and illustrates that the continuum coupling is not a dominant mechanism in explaining the position of the drip line of the oxygen isotopes. Thus, irrespective of whether we use a complex Gamow s.p. basis that defines a model space consisting of the $\left(1 s_{1 / 2}, i d_{3 / 2}\right)$ states (with $\left.i=0, \ldots, n_{\text {gamow }}\right)$, or a harmonic oscillator basis with the $\left(1 s_{1 / 2}, 0 d_{3 / 2}\right)$ states only, ${ }^{25} \mathrm{O}$ is unstable against particle emission as long as we use ${ }^{22} \mathrm{O}$ as a closed-shell core. This has important consequences for our theoretical understanding of the stability of matter. As pointed out in a recent calculation by Hagen et al 22], many-body forces such as three-body interactions are crucial in explaining the correct position of the drip lines. The fact that a calculation of ${ }^{25} \mathrm{O}$ with ${ }^{22} \mathrm{O}$ as a closed-shell core gives the correct qualitative description of the ground state of ${ }^{25} \mathrm{O}$, hints at the fact that with three valence neutrons only, missing many-body physics does not play a central role. This should be contrasted to realistic calculations that utilize ${ }^{16} \mathrm{O}$ as a closed core. With nine valence neutrons, ${ }^{25} \mathrm{O}$ is strongly overbound using the standard model space comprising the oscillator orbitals $\left(1 s_{1 / 2}, 0 d_{5 / 2}, 0 d_{3 / 2}\right)$ and a two-body effective interaction derived using MBPT. However, the location of the excited states in ${ }^{24} \mathrm{O}$ and the width of the ground state of ${ }^{25} \mathrm{O}$ depend crucially on the choice of basis, and we have shown that a GSM basis is needed in order to explain these states.

We conclude this work with an investigation of which parts of the NN interaction are crucial for an understanding of the above excited states in ${ }^{24} \mathrm{O}$. To achieve this, we single out the ${ }^{1} S_{0}$ partial wave component of the NN interaction and vary its strength. This partial wave is particularly important to our understanding of pairing correlations in nuclei. In order to investigate the importance of this partial wave on the lowlying resonance states in ${ }^{24} \mathrm{O}$, we vary the strength as $\tilde{V}(\alpha)=\alpha \times\langle S=0, L=0 ; J=0|V| S=0, L=0 ; J=0\rangle, \alpha \in[0,1]$ in a bare NN potential. Clearly, $\alpha=0$ corresponds to an $\mathrm{NN}$ interaction with no contribution from this partial wave while $\alpha=1$ corresponds to the original interaction. The low-lying resonance states are calculated for each $\tilde{V}(\alpha)$, and the results are shown in Fig. 3 One can clearly see how the ${ }^{1} S_{0}$ partial wave in the NN interaction influences the many-body resonances in ${ }^{24} \mathrm{O}$. The ground state gains additional binding when $\alpha$ increases since the ground state can be well described by the $\nu\left(1 s_{1 / 2}\right)^{2}$ configuration. At $\alpha=0$ the position of $1^{+}$ and $2^{+}$are interchanged with respect to experiment, and they are degenerate if one analyzes the real part of the resonance energies. When $\alpha$ increases to the full interaction strength, the $1^{+}-2^{+}$splitting increases to $530 \mathrm{keV}$, a result close to experiment. The ${ }^{1} S_{0}$ partial wave, a central contribution to pairing in nuclei, is thus crucial in order to explain correctly the low-lying resonant states in ${ }^{24} \mathrm{O}$.

Conclusions. We have described the low-lying states in 


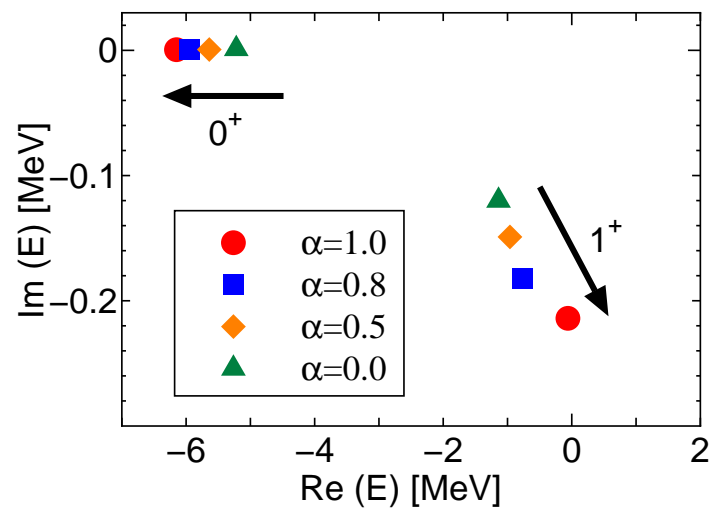

(a) The ground state and the $1^{+}$in ${ }^{24} \mathrm{O}$

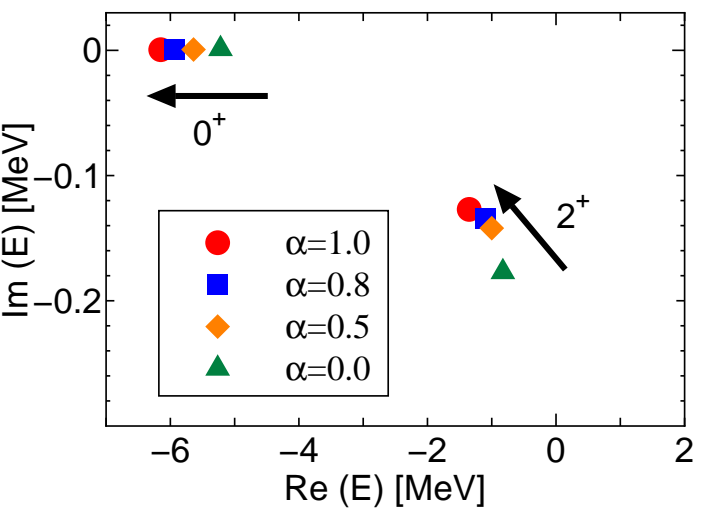

(b)The ground state and the $2^{+}$in ${ }^{24} \mathrm{O}$

FIG. 3: The energies of low-lying resonance states in ${ }^{24} \mathrm{O}$, as a function of the strength $\alpha$ of the ${ }^{1} S_{0}$ partial wave component of the nucleon-nucleon interaction. See text for further details.

the oxygen isotopes ${ }^{24} \mathrm{O}$ and ${ }^{25} \mathrm{O}$ within the framework of the GSM and effective interactions derived through many-body perturbation theory. We have shown that there is a fine interplay between many-body effects and the proximity of the continuum. We demonstrated that the inclusion of a discretized continuum and resonant states are crucial in order to describe the excited states of ${ }^{24} \mathrm{O}$ and the ground state of ${ }^{25} \mathrm{O}$. A Gamow basis is needed in order to reproduce both the correct excitation energies and widths of these states. In order to predict the location of the drip line, we found that a proper inclusion of many-body forces and correlation effects are the deciding factors. Furthermore, the ${ }^{1} S_{0}$ partial wave component of the NN interaction, an important contribution to strong pairing correlations in nuclei, plays an important role in explaining the correct value of these states. We conclude that the location of the drip line and excited states in the oxygen isotopes can only be determined by proper inclusion of both many-body correlations and the coupling with continuum degrees of freedom.

We thank Prof. T. Otsuka for valuable comments. This work has been supported in part by the JSPS coreto-core program, EFES and by a grant-in-aid for Scientific Research (A) 20244022, and by the Research Council of Norway (Supercomputing grant NN2977K). Oak Ridge National Laboratory is managed by UT-Batelle, LLC, for the U.S. Department of Energy under Contract No. DEAC05-00OR22725. KT thanks JSPS for a fellowship.
[1] N. Michel et al, J. Phys. G 36, 013101 (2009).

[2] A. Volya and V. Zelevinsky, Phys. Rev. C 74, 064314 (2006).

[3] J. Rotureau et al, Phys. Rev. Lett. 97, 110603 (2006).

[4] G. Hagen et al, Phys. Lett. B, 656 (4), 169-173, (2007).

[5] R. B. Wiringa, Steven C. Pieper, Phys. Rev. Lett. 89, 182501 (2002).

[6] P. Navratil and W. E. Ormand, Phys. Rev. Lett. 88, 152502 (2002).

[7] J. P. Schiffer Phys. Rev. Lett. 92, 162501 (2004).

[8] D. Warner, Nature 430, 517 (2004).

[9] T. Otsuka et al, Phys. Rev. Lett. 95, 232502 (2005).

[10] T. Otsuka et al, Phys. Rev. Lett. 87, 082502 (2001).

[11] Z. Elekes et al, Phys. Rev. Lett. 98, 102502 (2007).

[12] A. Schiller et al, Phys. Rev. Lett. 98, 102502 (2007).

[13] C. R. Hoffman et al, Phys. Rev. Lett. 100, 152502 (2008).

[14] P. G. Thirolf et al, Phys. Lett. B485, 16 (2000).

[15] M. Stanoiu et al, Phys. Rev. C 69, 034312 (2004).

[16] C. R. Hoffman et al, Phys. Lett. B672, 17 (2009).

[17] H. Sakurai et al., Phys. Lett. B 448, 180 (1999).

[18] B. A. Brown and W. A. Richter, Phys. Rev. C 72, 057301 (2005).
[19] B. A. Brown and W. A. Richter, Phys. Rev. C 74, 034315 (2006).

[20] M. Hjorth-Jensen, T. T. S. Kuo, and E. Osnes, Phys. Rep. 261, 125 (1995).

[21] L. Coraggio et al, Phys. Rev. C 75, 024311 (2007).

[22] G. Hagen et al, Phys. Rev. 80, 021306(R) (2009).

[23] Takaharu Otsuka et al, arXiv:0908.2607 (2009).

[24] Sofia Quaglioni and Petr Navratil, Phys. Rev. Lett. 101, 092501 (2008).

[25] N. Michel et al, Phys. Rev. C 67, 054311 (2003).

[26] S. K. Bogner, T. T. S. Kuo and A. Schwenk, Phys. Rep. 386, 1 (2003)

[27] G. Hagen, M. Hjorth-Jensen, and N. Michel, Phys. Rev. C 73, 044314 (2006).

[28] T. Berggren, Nucl. Phys. A109, 265 (1968).

[29] D. R. Entem and R. Machleidt, Phys. Lett. B524, 93 (2002).

[30] D. R. Entem and R. Machleidt, Phys. Rev. C 68, 41001 (2003).

[31] G. Audi, A. H. Wapstra and C. Thibault, Nucl. Phys. A729, 337 (2003). 